\title{
The Leaky Integrate-and-Fire Neuron: A Platform for Synaptic Model Exploration on the SpiNNaker Chip
}

\author{
A. D. Rast, F. Galluppi, X. Jin and S.B. Furber
}

\begin{abstract}
Large-scale neural hardware systems are trending increasingly towards the "neuromimetic" architecture: a general-purpose platform that specialises the hardware for neural networks but allows flexibility in model choice. Since the model is not hard-wired into the chip, exploration of different neural and synaptic models is not merely possible but provides a rich field for research: the possibility to use the hardware to establish useful abstractions of biological neural dynamics that could lead to a functional model of neural computation. Two areas of neural modelling stand out as central: 1) What level of detail in the neurodynamic model is necessary to achieve biologically realistic behaviour? 2) What is role and effect of different types of synapses in the computation? Using a universal event-driven neural chip, SpiNNaker, we develop a simple model, the Leaky-Integrate-and-Fire (LIF) neuron, as a tool for exploring the second of these questions, complementary to the existing Izhikevich model which allows exploration of the first of these questions. The LIF model permits the development of multiple synaptic models including fast AMPA/GABA-A synapses with or without STDP learning, and slow NMDA synapses, spanning a range of different dynamic time constants. Its simple dynamics make it possible to expand the complexity of synaptic response, while the general-purpose design of SpiNNaker makes it possible if necessary to increase the neurodynamic accuracy with Izhikevich (or even HodgkinHuxley) neurons with some tradeoff in model size. Furthermore, the LIF model is a universally-accepted "standard" neural model that provides a good basis for comparisons with software simulations and introduces minimal risk of obscuring important synaptic effects due to unusual neurodynamics. The simple models run thus far demonstrate the viability of both the LIF model and of various possible synaptic models on SpiNNaker and illustrate how it can be used as a platform for model exploration. Such an architecture provides a scalable system for high-performance large-scale neural modelling with complete freedom in model choice.
\end{abstract}

\section{Universal Neural Hardware: The NeEd for MODEL LIBRARIES}

W HAT is the purpose of dedicated neural network hardware? In the past, the answer was easy: model acceleration. However, rapid progress in standard digital hardware often outpaced the development cycle for firstgeneration neural chips, so that by the time they were available software simulators could outperform them anyway [1]. FPGA implementations offered hardware-like performance gains with greatly reduced design time [2], and added a new dimension: the ability to implement different models on the same platform and explore various architectural tradeoffs [3]. However, power and routability limitations with FPGA's effectively restricted their role to model prototyping [4]. Today,

The authors are with the School of Computer Science, The University of Manchester, Manchester, UK (email: \{rasta\}@cs.man.ac.uk).

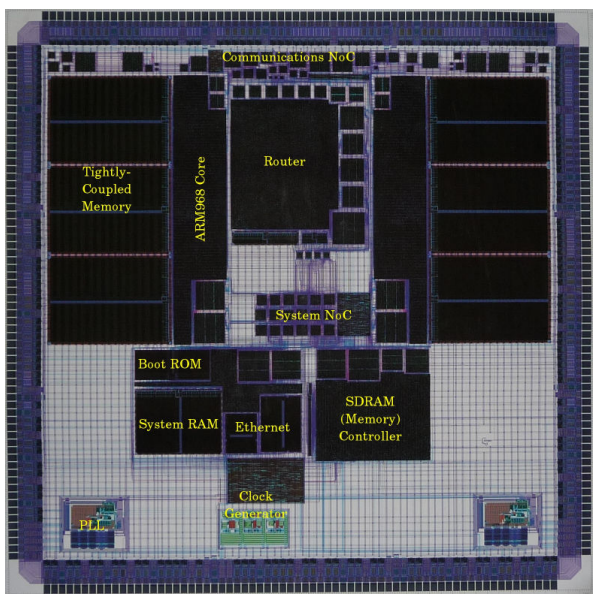

Fig. 1. SpiNNaker test chip

however, the rate of progress in standard digital hardware is flattening out, and the configurable-model concept that the FPGA introduced has given rise to new neural devices that offer various levels of model configurability without the scalability problems of the FPGA: "neuromimetic" chips [5]. These chips alter the nature of the research question: now it is not simply a matter of how to scale a neural model to large sizes but what model to scale.

The question of which neural network models are the "right" ones depends rather critically on what the purpose of the model is. A "one-size-fits-all" hardware neural model is thus unrealistic and somewhat irrelevant. Different research groups, with different goals, may want to use the hardware in different ways, and it is unrealistic to expect them to have, or even to acquire, the low-level hardware familiarity necessary to develop efficient models for universal neuromimetic chips. Configuring such chips can involve careful optimisation: the mapping of model to chip may be unusual and require intimate hardware knowledge [6]. Rather, the hardware developers should provide a library of different reference models, giving experimenters at minimum some models to get started with, and more generally a reference template that third-party developers could use to develop still further models and expand the hardware's model support. The beginnings of a model library are therefore a crucial step in the adoption of neuromimetic hardware for neural simulation.

With the SpiNNaker chip (fig. 1), we have introduced a neuromimetic platform for large-scale neural modelling ideal for exploring the effects of different models. Previously, 


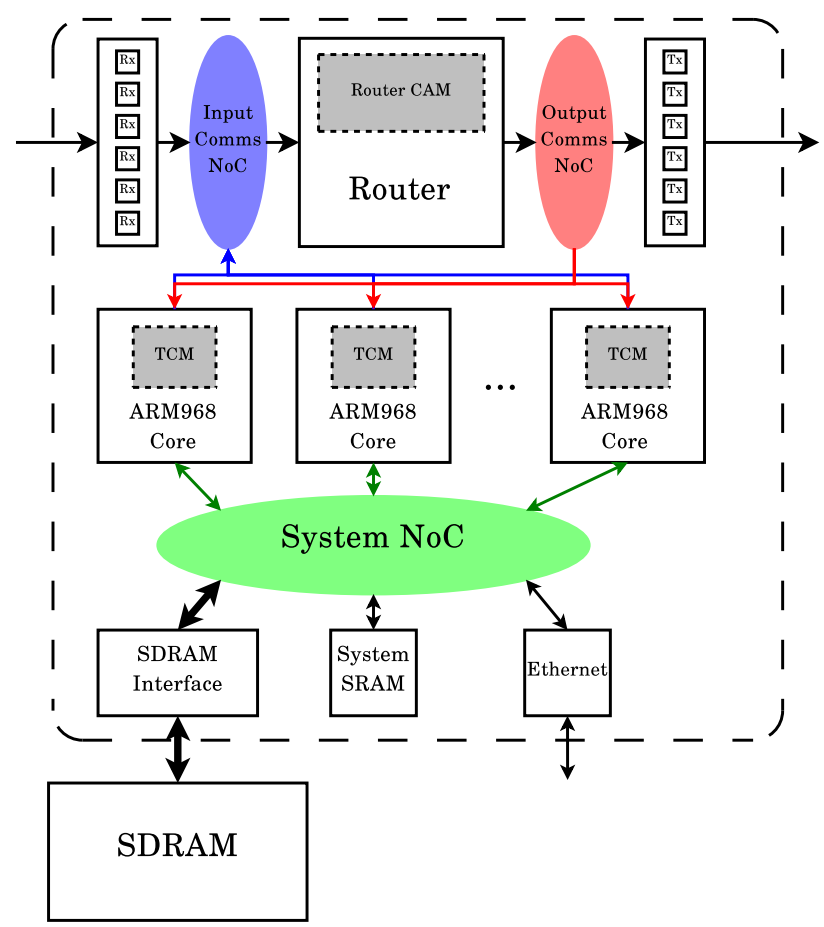

Fig. 2. SpiNNaker Architecture. The dashed box indicates the extent of the SpiNNaker chip. Dotted grey boxes indicate local memory areas.

we have introduced basic models for neural [7] and synaptic [8], [9] dynamics that illustrate the basic functionality of the device. However, these models are illustrative rather than prescriptive; having developed them we now wish to introduce further models to build a neural library and provide further examples to third-party developers of how to configure SpiNNaker. We have focussed on an important, popular neural model - the leaky-integrate-and-fire (LIF) model, and used it as a platform to develop other synaptic models, notably an NMDA synapse with slow voltage-gated dynamics. These models introduce a variety of techniques not yet demonstrated in the original reference models, while using and extending important core techniques from those models that show their general nature. Developing different neural models is a useful way not only to extend hardware capabilities, but to establish principles of efficient computation - a basis for approaching the question of what abstractions of neural function are useful.

\section{The SpinNaker Asynchronous Event-Driven ARCHITECTURE}

The SpiNNaker chip (fig. 2) is a universal neuromimetic platform containing programmable processing blocks embedded in a configurable asynchronous interconnect. Three major goals have driven design decisions: complete system virtualisation - where possible components are generic and configurable; analogy to biology - components use the same model of computation as "real" neural networks, provided this does not compromise their universal capabilities; and offthe-shelf internals - where suitable predesigned components
("IP") already exist, the system uses them. The primary features of the architecture are:

\section{Native Parallelism:}

"Real" neural networks are massively parallel processors. Native parallelism is therefore basic to the neuromimetic architecture. There are multiple ( 2 in the present implementation, 20 in a forthcoming version) general-purpose ARM968 processors per device. Each processor has its own private subsystem containing various devices to support neural functionality: a communications controller that handles packet-based I/O traffic, a DMA controller that provides transparent access to synaptic data residing off-chip in a separate memory, and a Timer to generate fixed time steps where models need them. Each "processing node" operates completely independently, using only local information to control execution and operating asynchronously from other processing nodes.

\section{Event-Driven Processing:}

Biological neurons communicate primarily through spikes: short-duration impulses whose precise shape is usually considered immaterial. Correspondingly, SpiNNaker uses Address-Event Representation (AER) to transmit neural signals between processors over a configurable packet-switched asynchronous interconnect. AER is an emerging neural communication standard [10] that abstracts spikes from neurobiology into a single atomic event, transmitting only the address of the neuron that fired. SpiNNaker extends the basic AER standard with an optional 32-bit payload. This fabric implements the support infrastructure for the eventdriven model.

\section{Incoherent Memory:}

The notion of controlled shared access to a central memory store simply does not exist in biology; neurons update using purely local information. Thus any processor may modify any memory location it can access without notifying or synchronising with other processors. Each SpiNNaker processor has access to 2 primary memory resources: its own local "Tightly-Coupled Memory" (TCM) and a chip-wide Synchronous Dynamic Random Access Memory (SDRAM) device, neither of which require or have support for coherence mechanisms. The TCM is only accessible to its own processor and contains both the executing code (in the $32 \mathrm{~KB}$ "Instruction TCM" (ITCM)) and local variables (in the 64KB "Data TCM" (DTCM)). The SDRAM, private to each chip but global to its processors, contains synaptic data (and possibly other large data structures). The processor's DMA controller handles synaptic data transfer over a >1GByte/s external link, making the synapse appear virtually local to the processor by bringing it into DTCM 
when an incoming packet arrives [11]. Since all data a given processor accesses is effectively private, SpiNNaker needs no coherence checking.

\section{Incremental Reconfiguration:}

Biological neural networks are plastic: the physical topology changes during operation. Likewise, the structural configuration of neuromimetic hardware can change dynamically while the system is running. SpiNNaker uses a distributed routing subsystem to direct packets through the network. Each chip has a packet-switching router that can be reprogrammed in part or in full by changing the routing table, thus making it possible to reconfigure the model topology on the fly. Under the AER format, the router uses a source routing protocol to direct traffic. Previous work ([12], [13]) describes the design of and configuration procedure for this network: the "Comms NoC".

\section{LIF MODEL IMPLEMENTATION}

\section{A. Implementation Constraints}

Implementation of the LIF model must consider SpiNNaker's hardware architecture. In particular, the following details of the hardware act as model design constraints:

\section{Elementary mathematical operations only}

The ARM968 has basic add and subtract, logical, shift, and multiply operations, but does not have native support for division, transcendental functions, and other nonlinear computations. Therefore, the model must express its processing in terms of simple polynomial mathematical operations.

\section{2-bit fixed-point representation}

Similarly, the ARM968 has no floating-point unit. The model needs to translate any floating-point quantities into fixed-point numbers, while determining a position for the decimal point, hence assigning a fractional precision.

\section{Limited local memory}

SpiNNaker's individual processors have $64 \mathrm{k}$ data memory and $32 \mathrm{k}$ instruction memory each. This effectively prohibits having synaptic data local at all times and limits the number of parameters. Memory management must therefore attempt to store as much information as possible on a perneuron rather than per-synapse basis.

\section{Limited time to process a neuron}

To stay within the real-time update requirement, each neuron must be able to update its state in the time between external events. If a processor is modelling multiple neurons, this means updating at worst in $\frac{1}{N} R_{\max }$, where $\mathrm{N}$ is the number of neurons modelled and $R_{\text {max }}$ is the maximum event rate.

Synaptic data only available on input event

Because of the limited memory, SpiNNaker stores synaptic data off-chip and brings it to the local processor only when an input event arrives. Synapse processing must therefore be scheduled for a fixed time after the input, and can only depend on information knowable at the time the input arrived.

These constraints allow us to form a general specification for SpiNNaker neural models using a set of design guidelines in combination with an abstract processing model.

\section{B. Implementation Rules}

To meet SpiNNaker's hardware constraints with an efficient, accurate model we introduce a set of design rules that help to define the model implementation. These rules are indicative but not forcing, so that while models generally obey this pattern they can in specific details deviate from it.

\section{Defer event processing with annotated delays}

The deferred-event model [8] is a method to allow event reordering. Under this scheme we only perform minimal processing at the time of a given event, storing state information in such a way as to be available to a future event, so that processes can wait upon contingent future events. Future events thus trigger state update relevant to the current event.

\section{Solve differential equations using the Euler method}

The Euler method is the simplest general way to solve nonlinear differential equations. In it, the processor updates the equations using a small fixed time step $\delta t$, using the formula $X(t+\delta t)=X(t)+$ $\frac{d x}{d t}(t+\delta t)$. The time step is programmable (nominally $1 \mathrm{~ms}$ in our models), allowing modellers to select the time step to optimise the precision/timing margin tradeoff.

Represent most variables using 16-bit values

Various studies indicate that 16-bit precision is adequate for most neural models [14], [15]. Since the ARM contains efficient 16-bit operations it makes sense to conserve memory space and use 16-bit variables throughout. Intermediate values, however, may use 32 bits to avoid unnecessary precision loss.

Precompute constant parameters where possible

By an astute choice of representation, it is often possible to transform a set of parameters in a neural equation into a single parameter that can be precomputed and simplifies the computation remaining. For example, in the expression $x(t)=$ $A e^{k t}$, we can use the substitution $\log _{a} b=\frac{\log _{c} b}{\log _{c} a}$, choose 2 for $\mathrm{c}$ and arrive at $x(t)=A\left(2^{\left(\log _{2} e\right) k t}\right)$, which allows us to precompute a new constant $\lambda=k \log _{2} e$ and determine $\mathrm{x}$ with simple shift operations.

\section{Compute non-polynomial functions by lookup table}

Lookup tables provide a simple, and in fact the only general way of computing an arbitrary function. The ARM takes at most $2 \mathrm{~N}$ instructions to compute a LUT-based function with $\mathrm{N}$ variables. Memory utilisation is a concern: a 16-bit lookup table is impractical; however, 8- or 12-bit lookup tables 


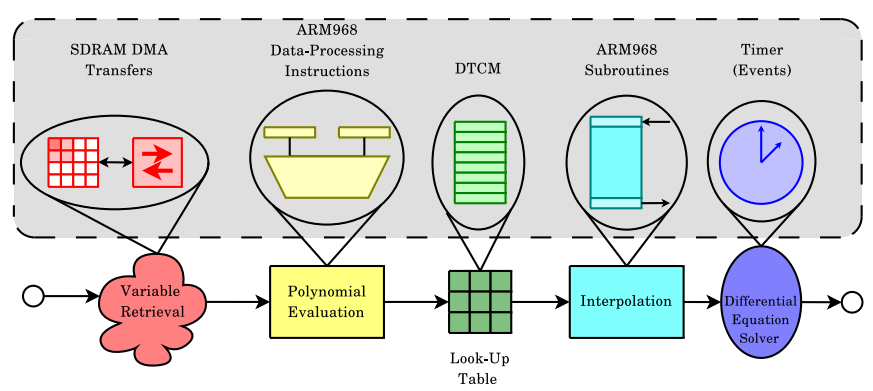

Fig. 3. A general event-driven function pipeline for neural networks. Variable retrieval recovers values stored from deferred-event processes as well as local values. Polynomial evaluation computes simple functions expressible as multiply and accumulate operations. These then can form the input to lookup table evaluation for more complex functions. Polynomial interpolation improves achieved precision where necessary, and then finally the differential equation solver can evaluate the expression (via Eulermethod integration). Each of these stages is optional (or evaluates to the identity function).

are feasible, occupying 512 or 8192 bytes for 16bit values respectively. Where models need greater precision we implement various interpolations.

\section{Exploit "free" operations such as shifting}

Most ARM instructions can execute conditionally, many can shift an operand before doing the instruction, and there are built-in multiply-accumulate instructions. Taking advantage of such "free" operations is an obvious optimisation.

Using these rules, we can build up a generalised function pipeline to represent a neural process that is adequate for most models (fig. 3).

\section{The LIF Model}

The LIF model uses this instruction pipeline. Many of the techniques it uses are common to the "reference" Izhikevich model we have described earlier [7]. The basic approach applies to virtually any spiking model with voltage-variable differential-equation dynamics: an illustration of the universal design of the software as well as the hardware. We will walk through the function pipeline stage by stage.

1) Variable Retrieval: Like the Izhikevich neuron, the LIF neuron uses the deferred-event model to place input spikes into a circular array of current "bins" representing the total input in a given time step (fig. 4). Arrival of an input triggers a DMA operation which retrieves a source neuron-indexed synaptic row. Completion of the DMA triggers a second stage of deferral, adding the synaptic weight (representing the current injection) to the bin corresponding to the synapse's delay. The Timer event that occurs when that delay expires recovers the total current from the bin and the neuron's associated state block, containing the voltage variable and internal parameters. We precompute natural frequency $f_{n}=$ $\frac{1}{\tau}$ from time constant $\tau$ in order to avoid division.

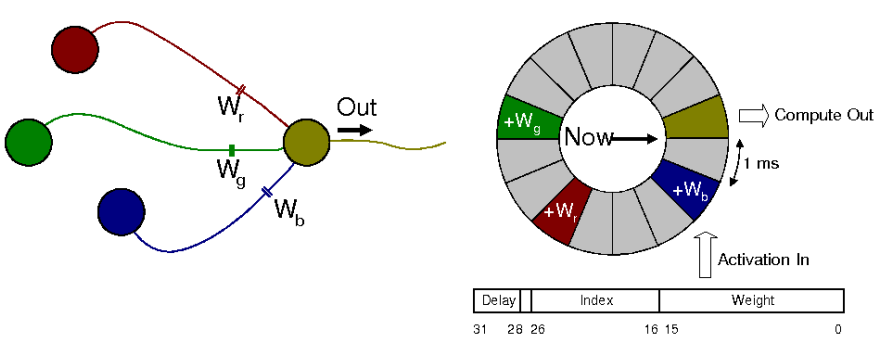

Fig. 4. SpiNNaker Neuron Binned Input Array. Inputs arrive into the bin corresponding to their respective delay. Output is computed from the bin pointed to by "Now".

2) Polynomial Evaluation: The basic LIF neuron equation is [16]

$$
\frac{d V}{d t}=f_{n}\left(V_{r}-V+I R\right)
$$

where $V_{r}$ is the rest voltage, I input current, $\mathrm{R}$ membrane resistance, and $\mathrm{V}$ membrane voltage. The right-hand side is a simple polynomial, which we can compute in 3 instructions. It is possible also to incorporate conductance-based synapses into this evaluation; the function is then

$$
\frac{d V}{d t}=f_{n}\left(V_{r}-V+T(t)\left(V-V_{n}\right)+I R\right)
$$

where $\mathrm{T}(\mathrm{t})$ is the synaptic "transmissivity", a precomputed value that incorporates the values of maximum synaptic conductance and specific membrane resistivity into the release probability. $V_{n}$ is the synaptic rest voltage.

3) Look-up table: For the "normal" LIF neuron there is no need for a lookup table function since the differential equation is polynomial. However, if we include conductancebased NMDA synapses, there is a voltage gating term [16]:

$$
G=\frac{1}{1+\frac{M g^{+}}{3.57} e^{\left(\frac{-V}{16.13}\right)}}
$$

where $\mathrm{Mg}^{+}$is the magnesium ion concentration. This function will use a (256-entry) LUT to compute its value.

4) Interpolation: Likewise in the standard LIF model there is no need for interpolation since the function can be computed exactly. If the model uses NMDA synapses, and if it requires better than 8-bit precision in the gating factor, however, we have implemented a spline-based interpolation. While efficient, it is not computationally free, requiring 48 cycles.

5) Differential Equation Solver: The Euler-method process evaluates the differential equation at each (1ms) Timer event. After evaluating the equation, it also tests whether the potential has exceeded the threshold $V_{t}$. If it has, it resets $\mathrm{V}$ to $V_{s}$. The LIF computation requires 10 instructions if the neuron does not fire (state update), 21 if the neuron fires (state update + spike generation), and 38 if the neuron fires and synapses have spike-timing-dependent plasticity (STDP) (state update + spike generation + STDP update). The high efficiency of this model makes it an ideal test-bed for exploring different synaptic models. 


\section{SynAPtiC Models}

Spiking neural networks can contain various different synapse types with different dynamics. At present the role of different synaptic types remains an area of intense research interest [17]. Equally significantly, the level of biophysical realism necessary to achieve useful behaviour or model actual brain dynamics is unclear. For instance, in the case of the well-known STDP plasticity rule, while many models exist describing the behaviour [18], [19], the actual biological data on STDP is noisy and of low accuracy. Observed STDP modifications exhibit a broad distribution for which the nominal functional form of STDP models usually constitute an envelope or upper bound to the modification [20]. This suggests that high repeatability or precision in STDP models is not particularly important.

SpiNNaker's completely virtual, general-purpose design makes it capable, in principle, of modelling synapses with biophysical realism down to the molecular level if necessary, but such high biological fidelity would almost certainly sacrifice real-time update speed and possibly require multiple processors to implement a single synapse. In view of observed synaptic variability, exact biological replication, or fidelity to a precise functional form, appears to be unnecessary for understanding their computational properties. This gives considerable latitude for experimenting with different synaptic models in order to investigate various tradeoffs between computational cost and functional accuracy. Using the LIF model gives not only a default "reference" model with known and easily tuned dynamics that expose the synaptic dynamics clearly, it also provides a very-low-computationalcost model, minimising machine cycles to allow more time for complex synaptic models. We are using the LIF model to test 2 different models.

The first model is a temporally accurate STDP model. Details of the technique and algorithm are in [8], [9]. This model demonstrates 2 important techniques: use of the deferred-event model to reorder processes, and exploiting the ARM's bit-shifting logic to maximise computational efficiency. Under the deferred-event model, the processor performs the synaptic update only upon receipt of a new presynaptic spike, using a pair of time stamps to record presynaptic and postsynaptic events so that the processor can track postsynaptic spikes happening after a given input. It can then retroactively apply the weight changes that would have happened to the synapse between the last input and the current input, using shift-and-add operations, before transmitting the spike through the synapse.

The second model adds N-Methyl-D-Aspartic (NMDA)mediated synapses exhibiting voltage gating with slow dynamics. This model presents 2 challenges: first, how to model the slow dynamics without having to retrieve the synaptic values multiple times, and second, how to implement the voltage gating process. 2 properties of NMDA synapses make it possible to implement an efficient algorithm. First, the synaptic current exhibits a linear kinetic, therefore instead of using sampled Euler-method evaluation it is possible to precompute the update for each input. Second, voltage gating is multiplicative and depends on the post-synaptic potential, thus the net contribution from all synapses with NMDA-mediated dynamics can be computed by multiplying their aggregate activation by the gating factor on a per (postsynaptic) neuron basis.

Precomputing the synapse open probability uses a variety of techniques. Since NMDA synaptic currents reach their maximum value quickly, usually within $1.5 \mathrm{~ms}$ of spike arrival, it is possible to neglect the effects of risetime dynamics, incorporating them into the delay. We can accommodate the slower decay dynamic by adding a second set of activation bins to each neuron that rotate every (slow time constant) $\tau \mathrm{ms}$. Precomputing the value to place in each bin after the initial $\left(T_{\max }\right)$ bin is trivial by exploiting the exponential property of shifting and using precomputed parameter scaling: shift the "weight" stored in the synaptic block, right by one place for each successive bin.

The complete process then functions as follows:

Presynaptic input event

1) Retrieve the synaptic row corresponding to the presynaptic neuron.

2) Calculate the effective bin delay (position of bin $P_{\max }$ ) using $T_{\max }=\delta_{w}\left(\frac{1}{\tau}\right)$, where $\delta_{w}$ is the real-time annotated delay in the synaptic weight.

3) Precompute the synaptic contribution (effective open probability) for each future bin.

4) Distribute the contributions into each bin by accumulating them with the current bin value.

\section{Neuron timer event}

1) Get the current system time $t_{\text {sys }}$.

2) Calculate whether the NMDA bin should rotate by computing $t_{\text {sys }} R E M \tau \leq \frac{1}{2 \tau}$.

3) If the bin should rotate, advance it and clear the previous bin.

4) Compute the gating factor by LUT.

5) Multiply the current NMDA bin by the gating factor.

6) Add to the (fast AMPA/GABA-A) activation.

The NMDA synaptic model takes advantage of the low instruction count of the LIF process. Updating the NMDA dynamics in a neuron requires 22 instructions. Thus the total instruction count rises only to 32 if the neuron does not fire, 43 if the neuron fires. This remains well within timing limits to permit $\sim 1000$ neurons per processor.

\section{Simulating Various Spiking Models on SPINNAKER}

\section{A. Single Neuron Dynamics}

To test the LIF model, we started with a single neuron. We tested single neuron dynamics by injecting short pulses of current into a neuron with the following parameters: $V_{0}=V_{s}=-75 m V, V_{r}=-66 m V, f_{n}=\frac{1}{4} m s^{-1}, R=8$, $V_{t}=-56 \mathrm{mV}$. To test the accuracy of our implementation we compared it with the same neuron implemented with Brian [21]. Results are in fig. 5: the difference in the spiking region is due to the fact that we artificially set $V=30 \mathrm{mV}$ 


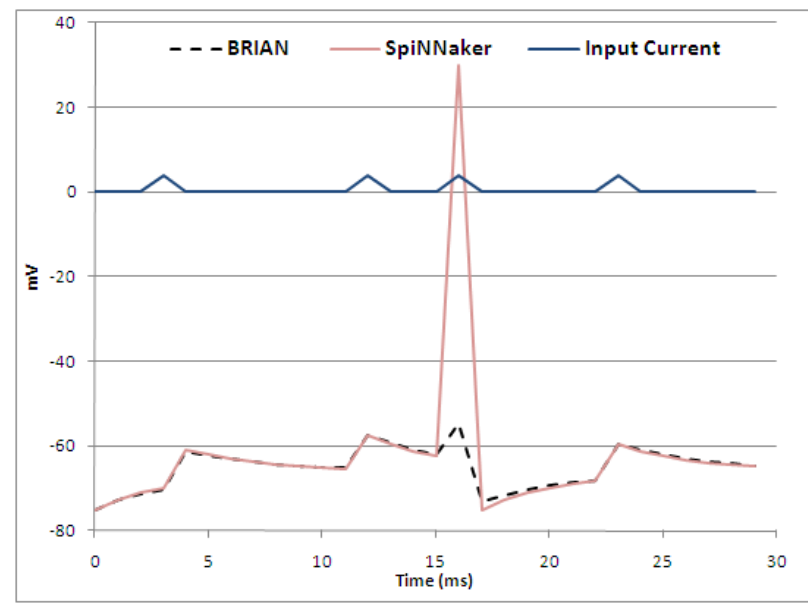

Fig. 5. Single neuron dynamics. We inject 4 pulses of current into the neuron. Traces compare the membrane potential of the simulation run on SpiNNaker (continuous line) with the same neuron implemented in Brian (dashed line).

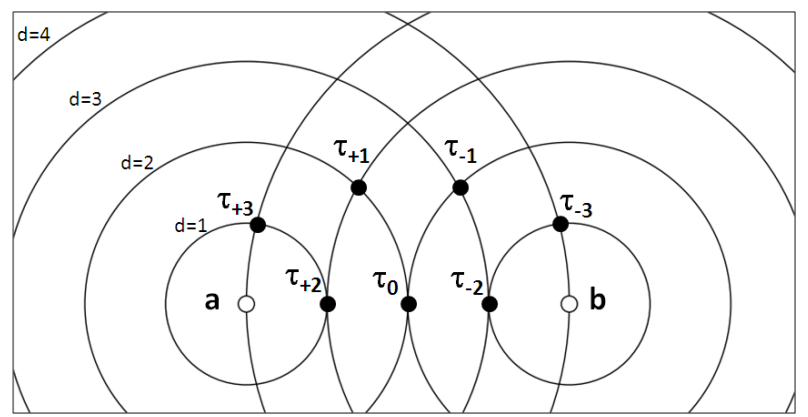

Fig. 6. Detection of the interpulse interval between spikes from neurons $a$ and $b . d$ denotes the synaptic delay between inputs and detectors. Detector neuron $\tau_{i}$ fires when the interpulse interval $t_{a}-t_{b}=i$.

when a neuron crosses the threshold in order to have a selfevident spike.

\section{B. Spike Propagation}

In order to test the time precision of spike generation and propagation processes we implemented a network capable of detecting inter-pulse interval between spikes generated by two neurons, reproducing the results in [22] (Section 2). Two input neurons connect to 7 detector neurons with a delay proportional to the distance (fig. 6).

The weights are set so that a detector will fire only if two coincident spikes arrive within a millisecond interval; detector neuron $\tau_{i}$ only fires when the inter-pulse interval $t_{a}-t_{b}=i$, where $t_{a}$ and $t_{b}$ are the absolute firing times of neurons $a$ and $b$ respectively. Fig. 7 represents the network structure, specifying the delays. Fig. 8 presents the simulation results. The network is able to discriminate the inter-pulse interval between the firings of neuron $a$ and neuron $b$ with millisecond precision by producing a spike from the corresponding detector neuron.

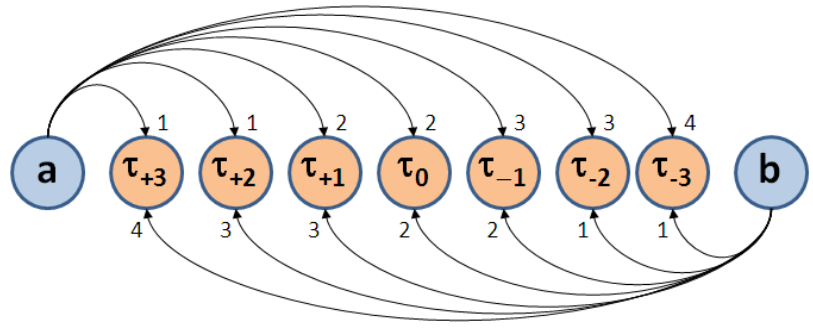

Fig. 7. Network Structure. Arrows represent connections between neurons. Values at the end of each arrow represent synaptic delays, set according to detector placement (cf. fig. 6).
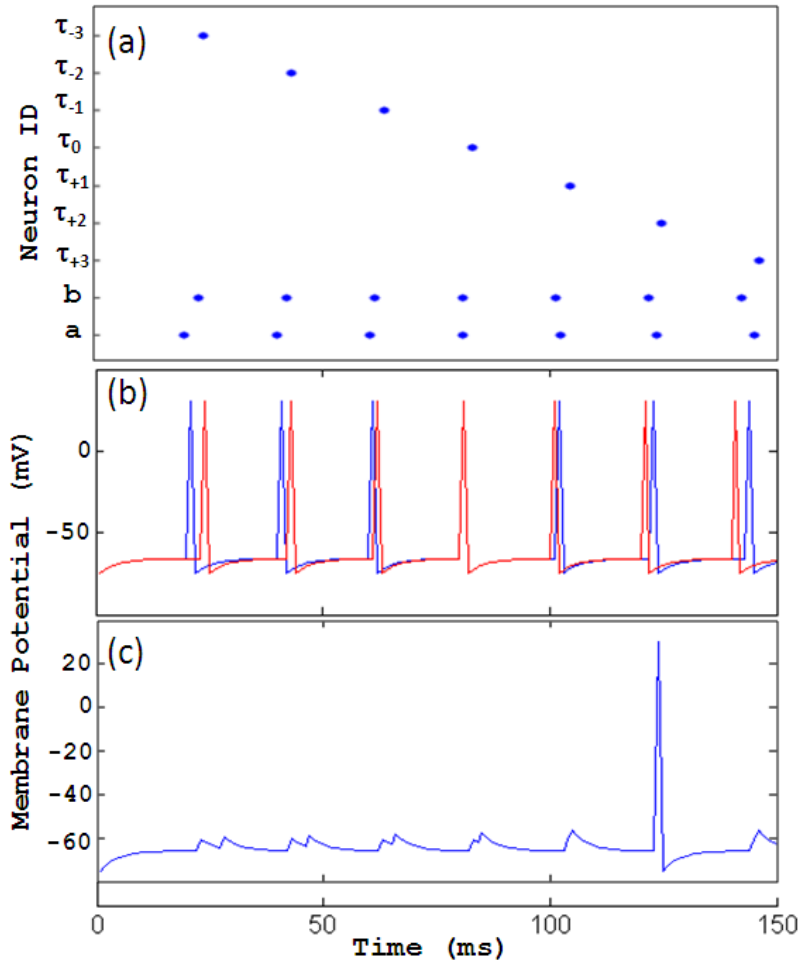

Fig. 8. Spike Propagation. (a) Raster Plot. Only the neuron detecting the correct interpulse interval fires. (b) Membrane potential of neurons a (blue) and $\mathrm{b}$ (red). (c) Membrane potential of detector neuron $\tau_{+2}$. The neuron only fires when spikes from neurons $\mathrm{a}$ and $\mathrm{b}$ converge on it with coherent synaptic delays.

\section{Oscillatory Network Activity}

We tested network dynamics by simulating the network in fig. 9: the network contains 100 neurons $\left(V_{0}=V s=\right.$ $-75 m V, V_{r}=-65 m V, f_{n}=\frac{1}{16} m s^{-1}, R=8, V_{t}=$ $-56 \mathrm{mV}$ ) divided into 80 excitatory neurons and 20 inhibitory neurons. Each neuron in the excitatory group connects to $56(70 \%)$ excitatory neurons and $2(10 \%)$ inhibitory neurons with random delay between 1 and $8 \mathrm{~ms}$. Each inhibitory neuron connects to every excitatory neuron with a delay of 1 or $2 \mathrm{~ms} .8$ neurons from the excitatory group form input neurons and receive a constant current injection of 3 $\mathrm{nA}$, making them fire approximately every $10 \mathrm{~ms}$. Excitatory weights are set in order to build up the background activity of the network slowly. Once there is sufficient activity, the 


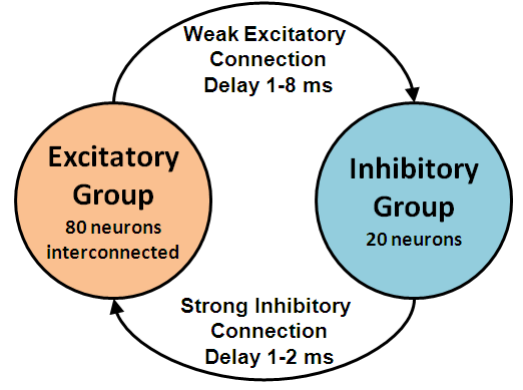

Fig. 9. Oscillatory Network Structure. Excitatory and inhibitory groups are connected so that when the activity of the excitatory group gets high the inhibitory group shuts down the activity of the whole network.

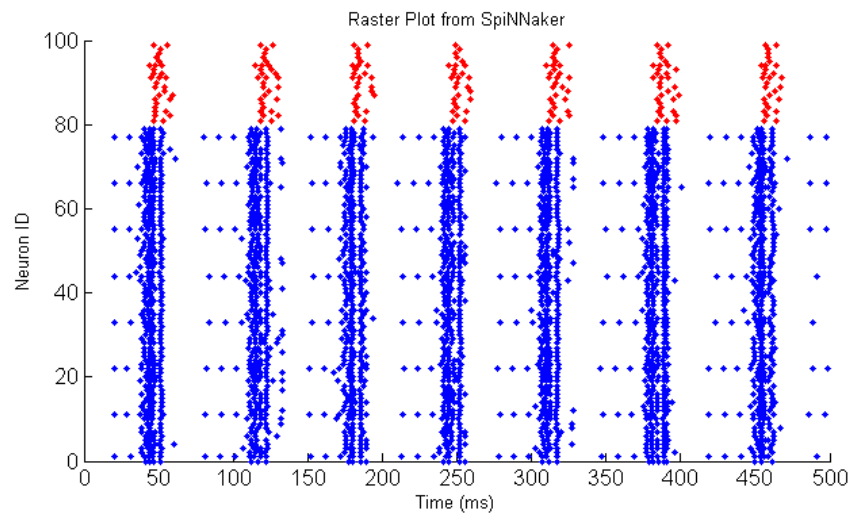

Fig. 10. Oscillatory Network Raster Plot. Input neurons (ID's 1, 11, 22, $33,44,55,66,77$ ) feed excitatory neurons (ID's 0-79), slowly building up the activity until excitatory neurons start to fire with high frequencies. Inhibitory neurons (ID 80-99) then activate, quenching the activity of the network.

whole excitatory group starts firing, causing the inhibitory neurons to fire a few ms later. Inhibitory weights are set to quench network activity quickly. Fig. 10 presents the results of the simulation.

\section{CONSIDERATIONS FOR EFFicient MOdELling LIBRARIES}

Implementing the LIF model has made it possible to consider efficiency limits within the SpiNNaker hardware. The core computation time of 10 instructions without spiking, 21 with, is probably the minimum possible for any model with broad acceptance within the spiking neural model community. Assuming a mean activity rate throughout the network of $1 \%$ (number of active neurons in a given processor), and providing for a timing margin of $0.2 \mathrm{~ms}$ in a $1 \mathrm{~ms}$ Timer (Euler sample) interval, we can examine the maximum number of neurons a single SpiNNaker processor could model (fig. 11).

For most models, memory capacity rather than processing overhead is the limiting factor: most configurations easily supported rates in excess of 2000 neurons/ms; considerably over the memory limit of 1724 AMPA/GABA-A-only neurons/processor, or 910 neurons with NMDA synapses. SDRAM external bandwidth turns out not to be a factor: 1 $\mathrm{GB} / \mathrm{s}$, even in worst-case conditions (100\% active synapses)

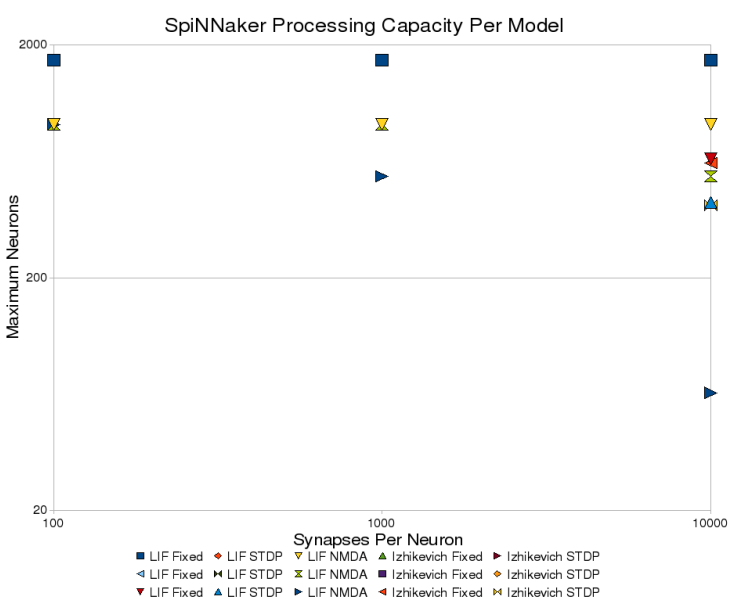

Fig. 11. Number of neurons per processor. The data considers neurons with 100,1000 , and 10,000 synapses with firing rates of $1 \mathrm{~Hz}, 10 \mathrm{~Hz}$, and $100 \mathrm{~Hz}$.

supports 12.8 updates/synapse/s with 1000 synapses/neuron. Of the various factors the mean spiking rate of the active population has the greatest impact on performance. This was particularly noticeable for NMDA synapses where high firing rates increase the number of iterations necessary to precompute future NMDA activation; a multiplicative effect. High rates also increase the mean activation, making it more probable that the NMDA gate will turn on, increasing the downstream firing rate. The result is a processing cascade and at the maximum firing rate of $100 \mathrm{~Hz}$ the number of neurons a processor can model drops drastically.

The LIF model can support more complex synaptic models that other spiking models such as the "reference" Izhikevich model or conductance-based models, simply because it has fewer instructions to perform. This in itself is a strong reason to make it the model of choice for large-scale studies of synaptic dynamics, but there is another and equally powerful motivation: ease of analysis.

Any research project must of necessity make a definite choice as to the scope of the research: what is being investigated. Unsurprisingly, the majority of investigations into new synaptic models have used the LIF neuron so as not to introduce too many experimental variables simultaneously. It is ideal because it is very simple, exhaustively analysed, and does not introduce complexities that might obscure the effects of a given synaptic model. Developing the LIF model for SpiNNaker adds it to the "neural library" so that modellers can insert the neuron as a "drop-in" component without having to spend any time developing or implementing the neurons themselves on SpiNNaker.

Implementing a function pipeline has given us a standard "template" for library components. It considers what functions SpiNNaker can implement efficiently. SpiNNaker's processors can easily compute polynomial functions but it is usually easier to implement other types, e.g. exponentials as a look-up table with polynomial interpolation. This adequately covers the right-hand-side of differential equations. 
For very simple cases it may be possible to solve such equations analytically, but for the general case, the Euler method evaluation we have used appears to be adequate. Creating a new component for the library is simply a matter of plugging in appropriate models for the pipeline stages, allowing for extensive software reuse because most of the support libraries, low-level utilities, and "housekeeping" code can be general across all models. Only the dynamics need change. The library therefore takes the form of a general infrastructure with model-specific core routines.

\section{CONCLUSIONS}

The LIF model we have created is an important reference model for developing new synaptic models, and has been instrumental in creating and refining the function pipeline, as a base standard for neural and synaptic libraries. Developing more models for these libraries on SpiNNaker is one of our major research foci. We are currently reworking the library into a series of $\mathrm{C}++$ template classes, where the template parameters can indicate the model type. This will further simplify future model development and provide a specification for third-party model building. More research on data precision and performance, particularly in comparison with "reference" LIF and other models is important and ongoing. The other major area of research is in increasing the scale of neural models. We are creating a large-scale neural model, based on the LIF neuron with AMPA/GABA-A and NMDA synapses, to simulate attentional control and response, with future development of short- and long-term memories. Such a system should form an effective, scalable demonstration network for a multichip SpiNNaker system.

In a larger context, the function pipeline model we developed may be a useful abstraction for neural hardware, regardless of platform. To create the function pipeline we attempted to decompose the general form of neurodynamic state equations into platform-neutral components that hardware can typically implement easily. Digital hardware can readily implement memories to form variable retrieval and LUT stages, and both analogue and digital hardware have effective blocks for polynomial evaluation and interpolation. Both DSP's and various analogue blocks offer efficient computation of differential equations. Thus one could build a neural system in building-block fashion, by chaining together various components using AER signalling, allowing for the construction of hybrid systems in addition to integrated approaches like SpiNNaker. More than anything else, SpiNNaker is valuable as a test bed for hardware architectures for neural models.

\section{ACKNOWLEDGEMENTS}

The Spinnaker project is supported by the Engineering and Physical Sciences Research Council, partly through the Advanced Processor Technologies Platform Partnership at the University of Manchester, and also by ARM and Silistix. Steve Furber holds a Royal Society-Wolfson Research Merit Award.

\section{REFERENCES}

[1] A.R.Omondi, "Neurocomputers: a dead end?" Int'l J. Neural Systems, vol. 10 , no. 6, pp. 475-481, Dec. 2000.

[2] D.Roggen, S.Hofmann, Y. Thoma, and D. Floreano, "Hardware spiking neural network with run-time reconfigurabile connectivity in an autonomous robot," in Proc. 2003 NASA/DoD Conf. Evolvable Hardware. IEEE Press, 2003, pp. 189-198.

[3] A.Upegui, C. Peńa-Reyes, and E.Sanchez, "An FPGA platform for online topology exploration of spiking neural networks," Microprocessors and Microsystems, vol. 29, no. 5, pp. 211-223, Jun. 2005.

[4] L. Maguire, T. M. McGinnity, B. Glackin, A. Ghani, A. Belatreche, and J. Harkin, "Challenges for large-scale implementations of spiking neural networks on FPGAs," Neurocomputing, vol. 71, Dec. 2007.

[5] S. Renaud, J. Tomas, Y. Bornat, A. Daouzli, and S. Saighi, "Neuromimetic ICs with Analog Cores: an Alternative for Spiking Neural Networks," in Proc. 2007 IEEE Int'l Symp. Circuits and Systems (ISCAS2007). IEEE Press, 2007.

[6] J. Fieres, J. Schemmel, and K. Meier, "Realizing biological spiking network models in a configurable wafer-scale hardware system," in Proc. 2008 Int'l Joint Conf. on Neural Networks (IJCNN2008). IEEE Press, 2008, pp. 969-976.

[7] X. Jin, S. Furber, and J. Woods, "Efficient modelling of spiking neural networks on a scalable chip multiprocessor," in Proc. 2008 Int'l Joint Conf. on Neural Networks (IJCNN2008), 2008.

[8] A. Rast, X. Jin, M. Khan, and S. Furber, "The deferred-event model for hardware-oriented spiking neural networks," in Proc. 2008 Int'l Conf. Neural Information Processing (ICONIP 2008), 2009.

[9] X. Jin, A. Rast, F. Galluppi, M. M. Khan, and S. Furber, "Implementing learning on the SpiNNaker universal neural chip multiprocessor," in Proc. 2009 Int'l Conf. Neural Information Processing (ICONIP 2009), 2009.

[10] D. Goldberg, G. Cauwenberghs, and A. Andreou, "Analog VLSI spiking neural network with address domain probabilistic synapses," in Proc. 2001 IEEE Int'l Symp. Circuits and Systems (ISCAS2001). IEEE Press, 2001, pp. 241-244.

[11] A. Rast, S. Yang, M. M. Khan, and S. Furber, "Virtual synaptic interconnect using an asynchronous network-on-chip," in Proc. 2008 Int'l Joint Conf. on Neural Networks (IJCNN2008), 2008.

[12] M. M. Khan, D. Lester, L. Plana, A. Rast, X. Jin, E. Painkras, and S. Furber, "SpiNNaker: Mapping neural networks onto a massivelyparallel chip multiprocessor," in Proc. 2008 Int'l Joint Conf. on Neural Networks (IJCNN2008), 2008.

[13] A. Brown, D. Lester, L. Plana, S. Furber, and P. Wilson, "SpiNNaker: The design automation problem," in Proc. 2008 Int'l Conf. Neural Information Processing (ICONIP 2008). Springer-Verlag, 2009.

[14] T. Daud, T. Duong, M. Tran, H. Langenbacher, and A. Thakoor, "High resolution synaptic weights and hardware-in-the-loop learning," in Proc. SPIE, vol. 2424, 1995, pp. 489-500.

[15] H.-P. Wang, E. Chicca, G. Indiveri, and T. J. Sejnowski, "Reliable computation in noisy backgrounds using real-time neuromorphic hardware," in Proc. 2007 IEEE Biomedical Circuits and Systems Conf. (BIOCAS2007), 2008, pp. 71-34.

[16] P. Dayan and L. Abbott, Theoretical Neuroscience. Cambridge: MIT Press, 2001.

[17] D. Durstewitz, "Implications of synaptic biophysics for recurrent network dynamics and active memory," Neural Networks, vol. 22, no. 8, pp. 1189-1200, Oct. 2009.

[18] H. Z. Shouval, M. F. Bear, and L. N. Cooper, "A unified model of NMDA receptor-dependent bidirectional synaptic plasticity," Proc. Nat. Acad. Sci. USA, vol. 99, no. 16, pp. 10 831-10 836, Aug. 2002.

[19] M. Hartley, N. Taylor, and J. Taylor, "Understanding spike-timedependent plasticity: A biologically motivated computational model," Neurocomputing, vol. 69, no. 16, pp. 2005-2016, Jul. 2006.

[20] G. Bi and M. Poo, "Synaptic Modifications in Cultured Hippocampal Neurons: Dependence on Spike Timing, Synaptic Strength, and Postsynaptic Cell Type," J. Neurosci., vol. 18, no. 24, pp. 10464-10472, Dec. 1998.

[21] D. Goodman and R. Brette, "Brian: a simulator for spiking neural networks in Python," Frontiers in Neuroinformatics, vol. 2, no. 5, Nov 2008.

[22] E. M. Izhikevich and F. Hoppensteadt, "Polychronous wavefront computations," International Journal of Bifurcation and Chaos, vol. 19, pp. 1733-1739, 2009. 